recommends itself for general use in studies where uniform responding over longer $\mathrm{CS}$ presentations is desired and where rate measures are more critical than latency measures.

The data on the acceleration ratios in relation to UCS intensity are presented in Fig. 1.

A repeated measures analysis of variance (Winer, 1962) indicated that the shock intensity variable had a highly significant effect on the acceleration ratios ( $F=24.41$, $\mathrm{p}<.001$, $\mathrm{df}=3,9$ ). In addition, a trend analysis showed that the relationship between UCS intensity and the acceleration ratios was strongly monotonic, the $\mathrm{F}$ ratios being $71.47, .24$, and 1.47 for the linear, quadratic, and cubic trend components, respectively. Furthermore, application of the Omega test (Hays, 1963) to the data demonstrated that the shock intensity variable accounted for $84 \%$ of the within-S variance.

In addition, the nearly identical performances of $S 6$ for two exposures at $1.25 \mathrm{~mA}$, separated by 2 months with intervening exposure to two other intensities, add weight to the stability criterion used here as a fair index of terminal performance. The fact that these two performances $(.542$ and .543) were preceded by exposure to the highest and lowest intensities, while not ruling out ordering effects, at least suggests that such effects either adapt out during the 5 days preceding testing or that they affect only preterminal performance.

\section{DISCUSSION}

The results of the present study seem to contrast with the findings of the previous Martin \& Riess (in press) study in that the magnitude of the ratios at the two comparable intensities was considerably greater in the first study (at .25, the mean ratio was .552 in the first study and .530 in this one. At 4.9, the mean ratio was .632 in the first study and .562 in the second.) These differences in absolute magnitude of the ratios appear to be a function of whether the avoidance contingencies are in effect or inoperative. In the initial study, testing was conducted under extinction conditions. A common occurrence under these conditions was for a CS presentation to be superimposed on a baseline of less than two responses per minute, the minimum required to avoid all shocks in the second study. The actual CS responding as such was thus more comparable for the two studies than the ratios suggest, owing to the artifact of depressed baselines obtained during extinction.

Secondly, Sidman, who first reported the phenomenon (Sidman, Herrnstein, \& Conrad, 1957) later reported (1960) that it eventually fails to occur. This contrasts with present results in that under the present conditions, the phenomenon was being reliably elicited in all Ss as late as 6 months following its initial occurrence and gave every indication of being indefinite. This discrepancy is predictable from Sidman's (1960) own account of the phenomenon. He considers acceleration a type of superstitious avoidance behavior. The fact that his procedure resulted in transitory increases only is attributed to the fact that shocks were included at the termination of the CS during avoidance, whereas in the present study they were included separately and omitted during avoidance. If a somewhat facetious distinction can be made between "successful" and "unsuccessful" superstitions, the impermanence of the phenomenon he reports is predictable from the fact that the increased avoidance rates failed to avoid the stimulus-contingent shock, rendering it in effect an extinction paradigm. In the present conditions, however, the stimulus-contingent shock was inoperative during avoidance; increased responding during the light successfully "avoided" the stimulus-contingent shock and the paradigm became a sort of spurious or S-defined avoidance procedure. Ss in the present study, then, were superstitiously reinforced for their avoidance increases by the shock omissions; in the Sidman et al procedure, this "reinforcement" failed to occur and acceleration eventually extinguished.

In this respect, conditioned acceleration is diametrically contrasted with conditioned suppression of appetitive responding. In the latter case, the phenomenon will continue indefinitely when the shocks are included during appetitive responding. It will eventually vanish if the Pavlovian reinforcement occurs separately and the shocks are excluded during appetitive responding. Precisely inverse relationships hold between inclusion vs exclusion of the Pavlovian contingencies and the permanence or impermanence of the phenomenon in conditioned acceleration.

\section{REFERENCES}

HAYES, W. L. Statistics for psychologists. New York: Holt, Rinehart, \& Winston, 1963.

KAMANO, D. K., \& ARP, D. J. Effects of chlordiazepoxide (Librium) on the acquisition and extinction of avoidance responses. Psychopharmacologia (Berlin), 1964, 6, 112-119.

MARTIN, L., \& RIESS, D. Effects of UCS intensity during preliminary discrete delay conditioning on conditioned acceleration during avoidance extinction. Journal of Comparative \& Physiological Psychology, in press.

RESCORLA, R. A. Inhibition of delay in Pavlovian fear conditioning. Journal of Comparative \& Physiological Psychology, 1967, 64, 114-120.

SIDMAN, M. Tactics of scientific research. New York: Basic Books, 1960a.

SIDMAN, M. Normal sources of pathological behavior. Science, 1960b, 132, 61-68.

SIDMAN, M., HERR NSTEIN, R. J., \& CONRAD, D. G. Maintenance of avoidance behavior by unavoidable shocks. Journal of Comparative \& Physiological Psychology, 1957, 50, 553-557. WINER, B. J. Statistical principles in experimental design. New York: MoGraw-Hill, 1962.

\title{
Discriminative classical conditioning of excitement anticipatory to food reward
}

EDWARD ZAMBLE, Queen's University at Kingston, Ont., Canada, and C. RICHARD KIRKWOOD, University of Washington Medical School, Seattle, Wash. 98105

Rats were trained with a discriminative Pavlovian paradigm while activity was measured. As in previous studies, an increase in activity occurred to CSt, but it was also found that activity wasless during $C S$ - than during a randomly unpaired stimulus. Contacts at the foodcup were also measured, and found to occur during CSt, mostly at times of least activity.
Pairing of a signal with noncontingent delivery of food leads to increased activity during the signal (Sheffield \& Campbell, 1954). However, the effects of a CS- have not been demonstrated.

It has been argued (e.g., Zamble, 1967) that the increased activity to a CS+ reveals a Pavlovian process which underlies the working of reinforcement in instrumental reward situations. Similarly, it is possible that responses to discriminative stimuli for nonreward may be mediated by inhibition of excitement by a Pavlovian CS-. Thus the present study incorporated a discriminative classical conditioning procedure in order to assess its effects on activity. 


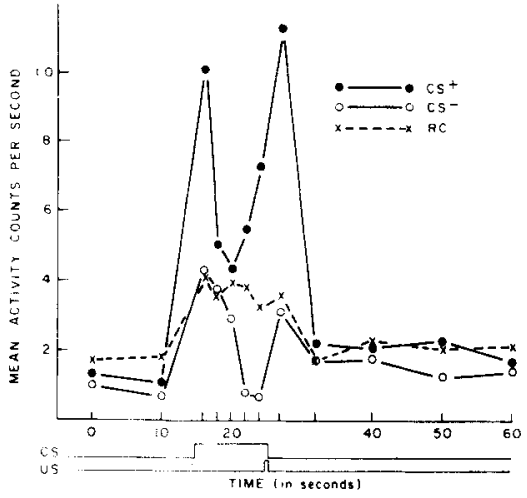

Fig. 1. Average activity during trials on the last training day (Day 6).

In addition, data were collected to provide information about behavior at the foodcup. Occurring just before the actual receipt of reward, the foodcup approach response is probably the behavior most susceptible to adventitious instrumental control. Foodcup contacts were measured during $\mathrm{CS}+$ to evaluate the hypothesis that the recorded activity increase is produced by such adventitious contingencies.

\section{METHOD}

The Ss were nine Sprague-Dawley male rats, approximately 100 days old at the start of the experiment.

Training was carried out in a rectangular box $\left(5 \frac{1}{2} \times 91 / 2 \times 8\right.$ in.), painted flat grey, with a clear Plexiglas front. The box was mounted on an activity recording platform as described before (Zamble, 1967). Movements of the cage were transmitted to a speaker coil, and the resultant voltage was used to trigger digital pulses. Mounted at one end of the training box were two foodcups,

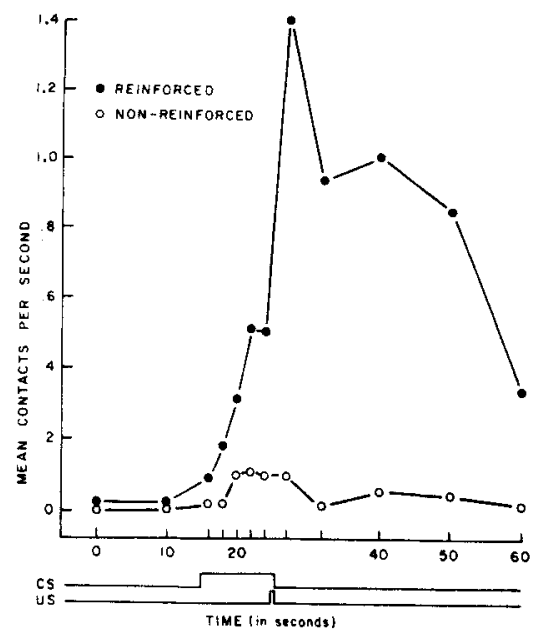

Fig. 2. Mean foodcup contacts during CS+ for the CC group on the last training day. connected to contact recorders, with sensitivity adjusted to record S's touching the foodcup with nose, feet, or mouth.

The entire apparatus was housed in a semisoundproof chamber with a dim houselight (HL), and a blower mounted outside. Rewards consisted of 45-mg Noyes pellets delivered through a plastic tube from a feeder mounted above the chamber. Stimuli were a $600-\mathrm{Hz}$ sine wave tone, and a $6000-\mathrm{Hz}$ square wave, presented through a speaker mounted on the training box.

The Ss were deprived at least 10 days on a schedule of $14 \mathrm{~g}$ Purina chow daily. As pretraining, they received a single 30 -min session in which pellets were delivered at random intervals to one of the foodcups (arbitrarily chosen for each S) in order to train them to approach the foodcup on food delivery.

There were two conditions, with four Ss in the classical conditioning group (CC) and five $S s$ in the random control group (RC). On each training day, $S$ was placed in the apparatus for a 5 -min habituation period, after which the HL was turned on and training begun. Each trial consisted of a presentation of one of the tones for $11 \mathrm{sec}$.

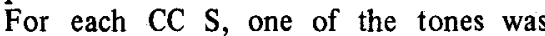
randomly chosen as CSt, and its onset was followed after $10 \mathrm{sec}$ by a single food pellet; the other tone (CS-) terminated without reinforcement. For RC Ss, both tones and food were delivered at the same frequency as for $\mathrm{CC}$, but the two were uncorrelated.

During each session, there were 20 presentations of each tone, randomly ordered at an average interval of $2 \mathrm{~min}$. At the end of each day's training, $S$ was removed to his home cage and fed $30 \mathrm{~min}$ later. There were 6 training days, for a total of 120 trials with each stimulus. Activity and responses on the foodcup were recorded continuously for $1 \mathrm{~min}$ on each trial, starting $20 \mathrm{sec}$ before the onset of the tone.

\section{RESULTS}

There were initially small increases in activity for both groups during both stimuli. Over the course of training, a difference developed for $\mathrm{CC}$ in the rates during $\mathrm{CS}+$ and CS-, as can be seen in Fig. 1, which shows data from the last training day. Differences in activity during the two stimuli did not approach significance for $\mathrm{RC}$, and the values have been combined. On Day 6, activity during CS+ was significantly greater than the combined values for the tones for $\mathrm{RC}$ $(\mathrm{t}=6.03, \mathrm{df}=7, \mathrm{p}<.01)$, and the latter was in turn significantly greater than the rate during CS $-(t=3.52$, df $=7, p<.01)$.

Contacts on both foodcups during the $\mathrm{CS}+$ (CC Ss only) on the last training day are shown in Fig. 2. Differences visible in Fig. 2 were not significant with a conventional $t$ statistic because of large individual differences, and instead, $t$ values for individual animals were combined using the Stouffer method. The greater number of contacts for the reinforced foodcup, as compared to the nonreinforced, is significant $(\mathfrak{I}=2.31, \quad n=4, \quad z=4.01$, $\mathrm{p}<.001$ ). On the reinforced foodcup there were significantly more contacts recorded during the last $4 \mathrm{sec}$ of $\mathrm{CS}+$ than during the first $4 \mathrm{sec}(\bar{t}=3.15, \quad n=4, \quad z=6.30$, $\mathrm{p}<.001)$.

\section{DISCUSSION}

The Pavlovian cues in the $\mathrm{CC}$ condition did acquire discriminative control over activity. Rates for the unpaired RC group indicate the unconditioned effects of stimulus presentation; compared to this baseline, activity increased during $\mathrm{CS}+$ and decreased during $\mathrm{CS}-$. Thus, we may extend the conditioned excitement hypothesis to include an inhibitory process. It has been pointed out before (Zamble, 1968) that the conditioned excitement notion alone cannot explain all of the phenomena of instrumental performance. Use of both excitatory and inhibitory processes should, however, increase the range of prediction.

The present data also bear on the possibility that the observed activity increase is produced by adventitious reward. It would be difficult for this hypothesis to account for the inhibitory effects of CS-. The evidence from foodcup behavior, although far from definitive, also mitigate against the primary role of adventitious reward. As has been found before (Zamble, 1967), most of the activity during CS+ occurred near the beginning. In contrast, foodcup contacts occurred almost entirely at the end of the signal. Thus, it seems quite unlikely that the recorded activity could have been substantially produced by behavior at the foodcup. Of course, some other responses might be adventitiously established, but it seems simpler to interpret the present data as indeed the result of Pavlovian processes.

\section{REFERENCES}

SCHEFFIELD, F.'D., \& CAMPBELL, B. A. The role of experience in the "spontaneous" activity in hungry rats. Journal of Comparative \& Physiological Psychology, 1954, 47, 97-100.

ZAMBLE, E. Classical conditioning of excitement anticipatory to food reward. Journal of Comparative \& Physiological Psychology, 1967, 63, 526-529.

ZAMBLE, E. Clinical conditioning of excitement anticipat ory to food reward: Partial reinforcement. Psychonomic Science, 1968, 10, 115-116.

\section{NOTE}

1. This research was performed at Yale University and supported by National Science Foundation Grant GB-04404 to Frederick D. Sheffield. The first author was a NSF Predoctoral Fellow when this work was carried out. 